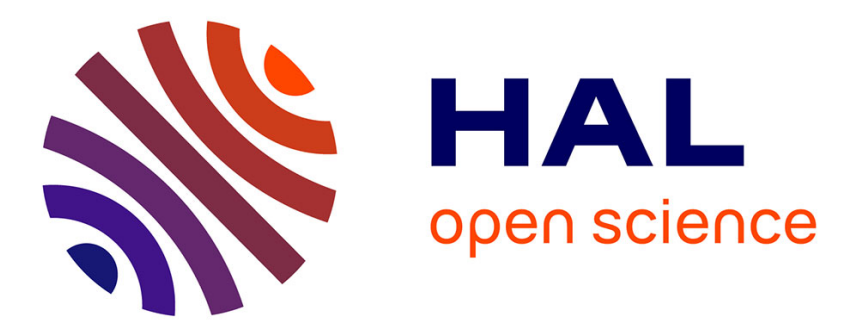

\title{
Probing 1D super-strongly correlated dipolar quantum
} gases

Roberta Citro, Stefania de Palo, Edmond Orignac, Paolo Pedri, Maria-Luisa Chiofalo

\section{- To cite this version:}

Roberta Citro, Stefania de Palo, Edmond Orignac, Paolo Pedri, Maria-Luisa Chiofalo. Probing 1D super-strongly correlated dipolar quantum gases. Laser Physics, 2009, 19 (4), pp.554-557. 10.1134/S1054660X09040045 . ensl-00517334

\section{HAL Id: ensl-00517334 \\ https://hal-ens-lyon.archives-ouvertes.fr/ensl-00517334}

Submitted on 14 Sep 2010

HAL is a multi-disciplinary open access archive for the deposit and dissemination of scientific research documents, whether they are published or not. The documents may come from teaching and research institutions in France or abroad, or from public or private research centers.
L'archive ouverte pluridisciplinaire HAL, est destinée au dépôt et à la diffusion de documents scientifiques de niveau recherche, publiés ou non, émanant des établissements d'enseignement et de recherche français ou étrangers, des laboratoires publics ou privés. 


\title{
Probing 1D super-strongly correlated dipolar quantum gases
}

\author{
R. Citro \\ Dipartimento di Fisica "E. R. Caianiello" and CNISM, Università degli Studi di Salerno, Salerno, Italy
}

S. De Palo

DEMOCRITOS INFM-CNR and Dipartimento di Fisica Teorica, Università Trieste, Trieste, Italy

E. Orignac

Laboratoire de Physique de l'École Normale Supérieure de Lyon, CNRS-UMR5672, Lyon, France

P. Pedri

Laboratoire de Physique Théorique de la Matière Condensée,

Université Pierre at Marie Curie, case courier 121, 4 place Jussieu, 75252 Paris Cedex, France Laboratoire de Physique des Lasers, CNRS UMR 7538,

Université Paris 13, 99 Avenue J.-B. Clément, 93430 Villetaneuse, France

\author{
M.-L. Chiofalo \\ INFN, Dpt. of Mathematics and Faculty of Pharmacy, University of Pisa, Pisa, Italy
}

\begin{abstract}
One-dimensional (1D) dipolar quantum gases are characterized by a very special condition where super-strong correlations occur to significantly affect the static and dynamical low-energy behavior. This behavior is accurately described by the Luttinger Liquid theory with parameter $K<1$. Dipolar Bose gases are routinely studied in laboratory with Chromium atoms. On the other hand, 1D realizations with molecular quantum gases can be at reach of current experimental expertises, allowing to explore such extreme quantum degenerate conditions which are the bottom line for designing technological devices.

Aim of the present contribution is to focus on the possible probes expected to signal the reach of Luttinger-Liquid behavior in 1D dipolar gases.
\end{abstract}

\section{INTRODUCTION}

One-dimensional (1D) dipolar quantum gases have been shown to always be in a very special condition where superstrong correlations occur to significantly affect the system behavior [1]. In this strongly-correlated regime, 1D dipolar gases are described by the Luttinger-Liquid theory with Luttinger parameter $K<1$, as we have demonstrated by comparing the results of Luttinger Liquid theory with Quantum Monte Carlo simulational data for static correlation functions. In fact, the dipolar tail of the interactions greatly enhances the correlation effects, which are also observable at the dynamical level [2].

Dipolar Bose gases are routinely realized in laboratory with Chromium atoms $[4,5]$ and more recently the possibility of reducing the effects of short range interactions with Fano-Feshbach resonances has been reported, so that a purely dipolar character emerges [6]. Moreover, 1D dipolar quantum gases may soon be within reach of current experimental expertises with molecular trapping. Orders of magnitudes provided in Ref. [1] suggest that molecular dipolar gases such as $\mathrm{SrO}$ [7] are promising candidates to explore the whole crossover from the Tonks-Girardeau regime with $K=1$ down to the quasi-ordered limit with $K \ll 1$.

The interest in realizing such extreme 1D quantum-degeneracy conditions accompanied by strong correlations is twofold. Such conditions would provide a real laboratory to test early theoretical predictions, and are the bottom line for designing technological devices for quantum information processing [8], precision measurements [9], and atomtronic devices $[10,11]$.

While for the detailed theoretical and numerical characterization of the physics of 1D dipolar gases we refer to our more recent review [3], the aim of the present contribution is to focus on the possible probes which are expected to signal the reach of Luttinger-Liquid behavior in 1D dipolar gases. After outlining the system under consideration in Sec. II, we then proceed to discuss in Secs. III,IV,V three different types of probes, two of them being already illustrated in detail in $[1,2,12]$. 


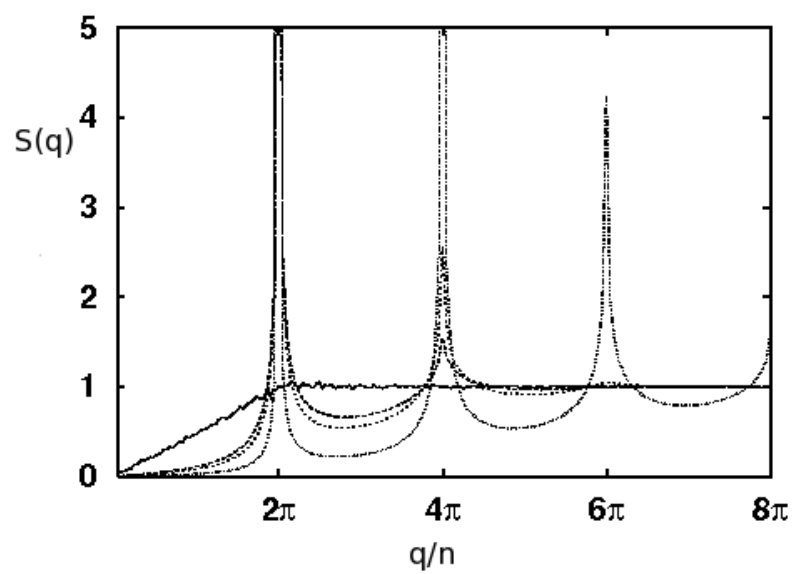

FIG. 1: $S(q)$ in dimensionless units for a dipolar gas with $N=40$ particles and different values of $n r_{0}=0.01,50,100$ and 1000 . Decreasing slopes as $q \rightarrow 0$ and the emergence of additional peaks correspond to increasing $n r_{0}$ values.

\section{THE SYSTEM}

The Hamiltonian modelling the system under consideration is that of $N$ atoms located at position $x_{i}$ with mass $M$ and permanent dipoles moments along and orthogonal to a line, yielding purely repulsive interactions:

$$
H=-\frac{1}{r_{s}^{2}} \sum_{i} \frac{\partial^{2}}{\partial x_{i}^{2}}+\frac{1}{r_{s}^{3}} \sum_{i<j} \frac{1}{\left|x_{i}-x_{j}\right|^{3}}
$$

in effective Rydberg units $R y^{*}=\hbar^{2} /\left(2 M r_{0}^{2}\right)$. The effective Bohr radius $r_{0} \equiv M C_{d d} /\left(2 \pi \hbar^{2}\right)$ is build up from the interaction strength $C_{d d}=\mu_{0} \mu_{d}^{2}$ for magnetic and $C_{d d}=d^{2} / \epsilon_{0}$ for electric dipoles. The interacting regime is governed by the usual parameter $r_{s}=1 /\left(n r_{0}\right)$ with $n$ the linear density, so that large densities correspond to strong correlations.

We study the ground-state properties as well as the static and dynamic low-energy behavior of this system by combining theoretical and numerical methods. These are in turn based on bosonization and Reptation Quamtum Monte Carlo (RQMC) techniques with finite number of particles, respectively. Details of the calculations can be found in Refs. $[1,2]$.

\section{STATIC PROBES}

One possible outcome of the RQMC numerical simulations is the static structure factor $S(k)$, which in terms of the density operator $\hat{n}$ is $S(q)=\int_{0}^{L} d x e^{-i q x}\langle\hat{n}(x) \hat{n}(0)\rangle$.

Fig. 1) [1] displays the overall structure at different densities values. Free fermion-like behavior as in the TonksGirardeau gas sets in at low density $\left(n r_{0}=0.01\right.$ in the graph). Quasi-Bragg peaks appear at reciprocal lattice vectors $q / n=2 \pi m$ with $m$ integer. Their number increases with increasing the density $n r_{0}$, until a quasi-ordered state sets in. Scaling studies of the RQMC data at different values of $N$ allow to conclude that the system is a Luttinger liquid.

In particular, a power-law decay of the peak heights in $S(q)$ and a divergence of their intensity with the system size is observed. In the Luttinger Liquid theory, this reflects into a flattening of the momentum distribution $n(q)$ as $n r_{0} \gg 1$. Both of these fingerprints of the Luttinger-liquid behavior can be probed in experiments with atomic gases. In particular, the static structure factor can be accessed by means of Bragg scattering techniques [13], while the momentum distribution $n(q)$ can be inferred from analysis of time-of-flight density images.

But the easiest way to probe the Luttinger liquid behavior in the whole crossover is to measure the eigenfrequencies of the collective trap modes, after modulating the trap by an external field with appropriate symmetry and as done in the very early seminal experiments with atomic gases [14, 15]. In Ref. [12] we have shown that for a system trapped in a shallow potential, an effective Luttinger-liquid hydrodynamic theory can be set up, discussing in detail the general equations and their range of validity and the results following the use of Local Density Approximation. Within this approximation we have determined the lowest collective modes of the harmonically trapped dipolar gas in the crossover. Figure 2 displays for example the frequencies of the lowest, breathing mode, which can be easily excited by periodically compressing the trap. This prediction enables e.g. the identification of the interaction regime of the trapped dipolar gas and provides a further testing of our theory. 


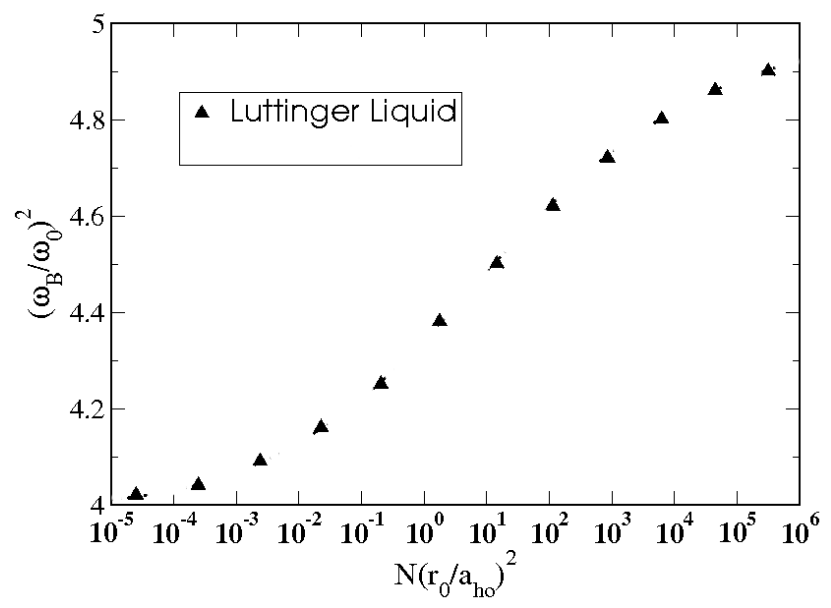

FIG. 2: Squared frequency $\omega_{B}^{2}$ of the breathing mode scaled to the trap frequency $\omega_{0} v s$. the interaction parameter $N\left(r_{0} / a_{\text {ho }}\right)^{2}$, as calculated from the Luttinger-liquid hydrodynamics (LL) [12].

\section{DYNAMICAL PROBES}

The energy of the low-lying excitations are extracted from the RQMC data for the imaginary-time density-density correlation function $F(q, \tau)$, after exploiting the Laplace transformation connecting $F(q, \tau)$ to the dynamical structure factor, that is $F(q, \tau)=\int_{0}^{\infty} d \omega \exp (-\omega \tau) S(q, \omega)$. After checking that different specific choices of $S(q, \omega)$ at zero temperature, corresponding to different approximations, do not lead to significant changes in the quantitative results, we assume the form

$$
S(q, \omega)=\sum_{i}^{\alpha} A_{i}(q) \delta\left(\omega-\omega_{i}\right)
$$

and within this choice $F(q, \tau)=\sum_{i=1, \alpha} A_{i}(q) e^{\omega_{i}(q) \tau}$, where $\omega_{i}$ is the excitation modes energy and $\alpha$ is the number of modes needed to yield the best $\chi^{2}$ in the fitting procedure, and depends on the values of $q$ and $n r_{0}$.

Fig. 3 displays the lowest excitation energies $\omega(q)$, while an accurate size effect analysis of the gap sizes as shown in Fig. 4 for $\omega(q / n=2 \pi)$ at different densities $n r_{0}=0.01,0.1,1,10$ and 1000, demonstrate a linear scaling $\omega_{N}(q=$ $2 \pi)=c\left(n r_{0}\right) / N$ with the slope $c\left(n r_{0}\right)$ an increasing function of $n r_{0}$. The overall result is that the gap is closed at all densities. This means that no roton gap appears at the first star of the reciprocal lattice $q=2 \pi$, implying the absence of superfluidity in this system, according to the Landau criterion. Once more, future experiments by means of e.g. Bragg spectroscopy techniques [13] may reveal this behavior, thereby providing a test for the present theory.

\section{INDUCED OPACITY BY A WEAK BARRIER}

We now turn to the third and last possible method to probe the occurrence of strongly repulsive Luttinger Liquid behavior in 1D dipolar gases, which exploits an earlier idea from Refs. [16] [17] [18] [19].

Let us consider a 1D Luttinger Liquid with parameters $u$ and $K$ and a weak local potential barrier with height $V_{0}$. The Hamiltonian of the system reads:

$$
H=\int \frac{d x}{2 \pi}\left[u K(\pi \Pi)^{2}+\frac{u}{K}\left(\partial_{x} \phi\right)^{2}\right]+\frac{V_{0}}{\pi \alpha} \int d x \sum_{m>0} A_{m} \cos 2 m \phi(0)
$$

where $\left[\phi(x), \Pi\left(x^{\prime}\right)\right]=i \delta\left(x-x^{\prime}\right), \alpha$ is a short distance cutoff, and $A_{m}$ are non-universal amplitudes. We define $V_{m}=V_{0} A_{m}$. The last term in Eq.(3) represents a backscattering operator in the usual fermionic language. A renormalization group calculation[16] shows that:

$$
\frac{d V_{m}}{d \ell}=\left(1-m^{2} K\right) V_{m}
$$




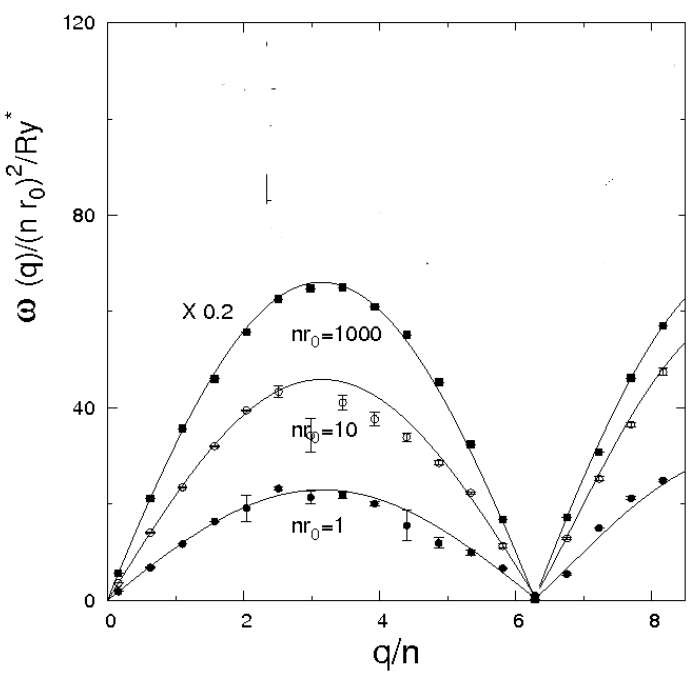

FIG. 3: Lowest excitation energies $\omega(q)$ in $R y^{*}$ units and scaled by $\left(n r_{0}\right)^{2}$, for a dipolar gas with $N=40$ and different values of $n r_{0}=1,10$ and 1000 as in the legend. Symbols with error bars are energies extracted using (2), the solid line is a guide to the eye. The curve at $n r_{0}=1000$ is depressed by a factor of 5 for graphical reasons.

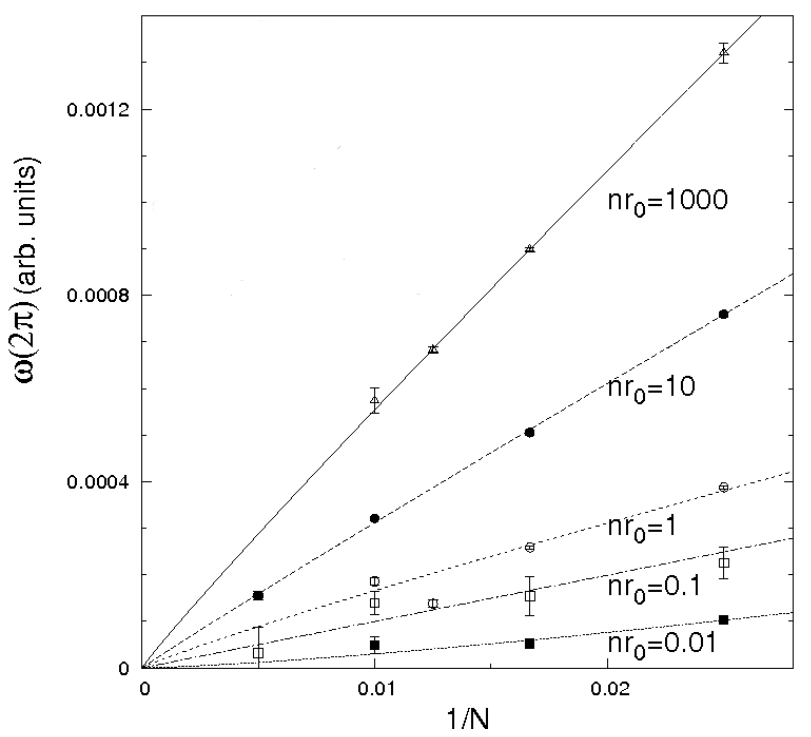

FIG. 4: $1 / N$ scaling of $\omega(q=2 \pi)$ in arbitrary units at $n r_{0}=0.01,0.1,1,10$ and 1000 as in the legend. Symbols with error bars: RQMC data. Solid lines: fit to the data.

where $\alpha(\ell)=\alpha e^{\ell}$ is the running cutoff and $\ell$ the length scale. For $K>1$, all the $V_{m}$ decay exponentially to zero, indicating that the barrier is becoming transparent. For $K<1, V_{1}$ is growing to infinity. This indicates that there is a strong coupling fixed point, where the phase field $\phi(0)$ is locked to the value of $\pi / 2$ in order to minimize the potential energy[16]. The boundary condition $\phi(0)=\pi / 2$ means that the system becomes equivalent to two independent semiinfinite Luttinger liquids[20], with total reflection of the particles on the boundary. The barrier is thus completely opaque for $K<1$. The case $K=1$ is marginal. Since for $K=1$ the system is equivalent to free fermions, it is easy to show that in that case there is a reflection and a transmission coefficient across the barrier.

One can thus imagine to push slowly a dipolar atomic beam along a 1D waveguide, against a barrier of appropriate height $V_{0}$ and simply observe whether the barrier is opaque or not to the passage of the beam. Since the dipolar gas always have $K<1$, the barrier should in fact always be opaque, in contrast with the case of a Lieb-Liniger gas, with pure contact interaction, for which $K>1$ and the barrier is transparent. The opacity of the weak barrier could be 
probed in experiments by standard absorption or yet in situ phase contrast imaging of the condensate[21].

\section{CONCLUSIONS}

In conclusion, we have discussed a few experimentally feasible methods which can be used to probe the occurence of super-strongly correlated behavior in 1D dipolar quantum gases. In particular, we have identified the fingerprints of such behavior in possible measurements of static and dynamic structure factors, of the momentum distribution, of the collective trap modes, and of the analog of the induced-transparency mechanism. Ongoing experiments with molecular dipolar gases may reveal one or more of these predictions.

\section{Acknowledgements}

P.P. acknowledges financial support from Ministère de l'Enseignement Supérieur et de la Recherche and IFRAF (Institut Francilien de Recherche sur les Atomes Froids).

[1] R. Citro, E. Orignac, S. D. Palo, and M. L. Chiofalo, Phys. Rev. A 75, 051602R (2007).

[2] S. De Palo, E. Orignac, R. Citro, and M. L. Chiofalo, Phys. Rev. B 77, 212101R (2008).

3] R. Citro, S. De Palo, E. Orignac, P. Pedri, and M. L. Chiofalo, New J. of Phys. 10, 45011 (2008).

[4] A. Griesmaier, J. Werner, S. Hensler, J. Stuhler, T. Pfau, Phys. Rev. Lett. 94, 160401 (2005).

[5] T. Lahaye, T. Koch, B. Fröhlich, M. Fattori, J. Metz, A. Griesmaier, S. Giovanazzi, and T. Pfau, Nature (London) 448, $672(2007)$.

[6] T. Lahaye, J. Metz, T. Koch, B. Fröhlich, A. Griesmaier, and T. Pfau, arXiv:0808.3876v1 (2008).

[7] P. Rabl and P. Zoller, Phys. Rev. A) 76, 42308 (2007).

[8] See e.g. I. Bloch, Nature Physics 123 (2005) and References therein.

[9] See e.g. M. A. Kasevich, Science 2981363 (2002).

[10] B. T. Seaman, M. Kramer, D. Z. Anderson, and M. J. Holland, Phys. Rev. A 75, 23615 (2007).

[11] M. L. Chiofalo, M. Artoni, and G. C. L. Rocca, New J. of Phys. 5, 78 (2003).

[12] P. Pedri, S. De Palo, E. Orignac, R. Citro, and M. L. Chiofalo, Phys. Rev. A 77, 15601 (2008).

[13] D. M. Stamper-Kurn, A. P. Chikkatur, A. Görlitz, S. Inouye, S. Gupta, D. E. Pritchard, and W. Ketterle, Phys. Rev. Lett. 83, 2876 (1999).

[14] D. S. Jin, M. R. Matthews, J. R. Ensher, et al., Phys. Rev. Lett. 78, 764 (1997).

[15] D. M. Stamper-Kurn, H. J. Miesner, S. Inouye, et al., Phys. Rev. Lett. 81, 500 (1998).

[16] C. Kane and M. P. A. Fisher, Phys. Rev. B 46, 15233 (1992).

[17] C. Kane and M. P. A. Fisher, Phys. Rev. Lett. 68, 1220 (1992).

[18] H. Saleur, in New Theoretical Approaches to Strongly Correlated systems, edited by A. M. Tsvelik (Kluwer Academic Publishers, Dordrecht, 2001), vol. 23 of NATO SCIENCE SERIES: II: Mathematics, Physics and Chemistry, chap. 3, p. 47.

[19] H. Saleur, in Topological aspects of low dimensional systems, edited by A. Comtet, T. Jolicoeur, S. Ouvry, and F. David (Springer, Berlin, 1998), vol. 69 of Les Houches Summer School, p. 475.

[20] M. Fabrizio and A. O. Gogolin, Phys. Rev. B 51, 17827 (1995).

[21] R. Onofrio, C. Raman, J. M. Vogels, J. R. Abo-Shaeer, A. P. Chikkatur, and W. Ketterle, Phys. Rev. Lett. 85, 2228 (2000). 